\title{
Coalition Formation and Trust in Collaborative Control
}

\author{
John S. Baras, Tao Jiang and Pedram Hovareshti \\ Department of Electrical and Computer Engineering \\ and Institute for Systems Research \\ University of Maryland \\ College Park, MD \\ \{baras, tjiang, hovareshp\}@umd.edu
}

\begin{abstract}
We consider the collaboration of autonomous agents as a coalitional game subject to constraints, like communication, trust or reputation. We analyze the tradeoff between the benefits of collaboration and these constraints (or costs of collaboration) via dynamic, iterative stochastic games. The agents can observe locally the actions of other agents in their neighborhood and decide whether to collaborate or not in a distributed asynchronous manner. Trust values are taken into consideration when agents act and collaborate. Specifically they affect the selection of agents to collaborate with. We consider only problems where the information used for either estimating reputations or deciding on strategies is mostly local; i.e. from the neighbors of each agent. We show that randomized algorithms emerge as the agents try to reach the maximum payoff. We also investigate the topology of the formed network by studying the second largest eigenvalue of the corresponding graph, and describe its effects on control performance.
\end{abstract}

\section{INTRODUCTION}

Dynamic networked systems are used as models for many phenomena and situations in science and engineering: communication networks, collaborating robots, organizations, societal systems and communities, economic systems and biological systems. Discovering fundamental principles governing the design-synthesis, control-operation, and performance evaluation of dynamic networked systems represents a major research challenge currently in science and engineering at large. The recent emphasis on and significance of this challenge is well described in the recent reports on Network Science [1], [2].

In this paper we develop the fundamental view that agents in such a network are dynamic entities that collaborate because via collaboration they can accomplish objectives and goals much better than working alone, or even accomplish objectives that they cannot achieve alone at all. Yet the benefits derived from such collaboration require some costs (or expenditures), for example due to communications, or due to energy expenditure. Or in equivalent terms, the collaboration is subject to constraints (static or dynamic). Understanding and quantifying this tradeoff between the benefits vs the costs of collaboration, leads to new methods that can be used to analyze, design and control/operate networks of agents. Multiple metrics for benefits and costs can be considered within this framework; that is we can consider vector valued benefits and costs of collaboration. Furthermore, we investigate topology effects of the formed networks. We study the topology efficiency while the benefitcost relations change.

This paper is organized as follows. Section II defines the graphic model and benefit-cost relation we use in the paper. A description of the coalition formation game is provided in Sec. III. Section IV investigates the dynamics of the game, including its convergence, steady state and topology at the steady state. Section V concludes the paper and discusses future work.

\section{PRoBlem Formulation}

\section{A. Graphic model}

Suppose there are $n$ nodes $^{1}$ in the network. Define the set of nodes $N=\{1,2, \ldots, n\}$. The communication structure of the network is represented by an undirected graph $g$, where a link between two nodes implies that they are able to directly communicate. It is natural to assume that nodes only agree to collaborate with those who are willing to collaborate. Thus a link in a network means that two end nodes agree to collaborate with each other. Notice that even though links are undirectional, the weights (related to gain and cost mentioned below) of the links are not undirectional. Suppose there is a link between node $i$ and $j$. The weights on link $i j$, denoted as $w_{i j}$ and $w_{j i}$ for two directions, are not necessary the same.

Let $g^{N}$ represent the complete graph, where every node is directly connected to every other node, and let the set $G=\left\{g \mid g \subseteq g^{N}\right\}$ be the set of all possible graphs. If $i$ and $j$ are directly linked in $g$, we write $i j \in g$. Let $g+i j$ denote the graph obtained by adding link $i j$ to the existing graph $g$ where $i j \notin g$ and $g-i j$ denote the graph obtained by severing link $i j$ from the existing graph $g$ where $i j \in g$ (i.e., $g+i j=g \cup\{i j\}$ and $g-i j=g \backslash\{i j\})$. The set of nodes in graph $g$ is $N(g)=\{i \mid i \in g\}$ and $n(g)$ is the number of nodes in $g$.

A communication link is established only if two end nodes agree to collaborate with each other, i.e., they are directly connected with each other in $g$. Once the link is added, two end nodes join one coalition and they agree to forward all the traffic from each other. Note that indirect communication between two players require that there is a path connecting them. A path in $g$ connecting $i_{1}$ and $i_{m}$ is

\footnotetext{
${ }^{1}$ In this paper, the terms node, player and agent are interchangeable.
} 
a set of distinct nodes $\left\{i_{1}, i_{2}, \ldots, i_{m}\right\} \subset N(g)$, such that $\left\{i_{1} i_{2}, i_{2} i_{3}, \ldots, i_{m-1} i_{m}\right\} \subset g$.

The communication structure $g$ gives rise to a partition of the node set into groups of nodes who can communicate with each other. A coalition of $g$ is a subgraph $g^{\prime} \subset g$, where $\forall i \in N\left(g^{\prime}\right)$ and $j \in N\left(g^{\prime}\right), i \neq j$, there is a path in $g^{\prime}$ connecting $i$ and $j$, and $i j \in g$ implies $i j \in g^{\prime}$.

\section{B. Benefit and cost}

As we have discussed, agents obtain benefits by joining a coalition. Suppose the characteristics of each node are represented as a profile. The profile of node $i$ is $x_{i}$, which includes characteristics of several aspects. For instance, in communication networks, $x_{i}$ can include the location of node $i$, neighbor set of node $i$, power level, noise, interference etc. Then both the benefit and the cost that occur when node $i$ connects with node $j$ is a function of $x_{i}$ and $x_{j}$. We denote by $B_{i j}$ the benefit node $i$ obtains by collaborating with node $j$. On the other hand, establishing links is costly; we denote the cost of activating link $i j$ by $C_{i j}$.

We are particularly interested in studying one type of cost or constraints: the trust values nodes have on others. The performance of autonomous agents is well-known to suffer from "free-riding" as there is a natural incentive for nodes to only consume, but not contribute to the services of the system. One well-known solution is the application of trust establishment and management systems. By trust we mean an estimate about an agent's actual quality in terms of its behavior in the network, which is sometimes also referred to as reputation. Nodes with a high trust value are then favored, while nodes with a low trust value are isolated from others. Assume the trust value node $i$ has on node $j$ is denoted as $s_{i j}$. The simple constraint will be that $i$ only establishes a direct collaboration with node $j$, if $s_{i j}>\theta$, i.e., a node with a trust value less than $\theta$ is untrusted.

In this paper, we will study coalition formation under trust constraints. There are many ways to incorporate trust constraints. One way used is the so-called 'hard constraint'. As long as $s_{i j}$ does not satisfy certain conditions, a link cannot be established at all between nodes $i$ and $j$. The threshold $\theta$ mentioned above is an example. Another way is the so-called 'soft constraint', where trust is considered as a risk of collaboration. The risk is the cost node $i$ expects to have when collaborating with node $j$ and is integrated into the cost $C_{i j}$. Our previous work on trust and cooperation [3] showed that both ways can be used as incentive for cooperation among selfish nodes. Trust values are based on local monitoring and previous experience. Notice that these trust values may change over time because new information has been received about agents, or agents' behavior has changed [4].

In the rest of this section, we describe examples of gains and costs in various networks:

\section{Wireless networks}

The benefit of nodes in wireless networks can be the rate of data flow they receive, which is known to be related to the received power. Let $x_{i}$ denote the physical coordinates of node $i$. Then we can define the benefit function $B_{i j}=$ $P_{j} l\left(x_{i}, x_{j}\right)$, where $P_{j}$ is the power spend to generate the transmission and $l\left(x_{i}, x_{j}\right)<1$ is the loss factor due to isotropic dispersion and absorption in the environment.

On the other hand, the power used in transmission is a cost. Therefore, the cost for node $i$ to activate his communication link to node $j$ can be equal to the transmission energy (or power) necessary for $i$ to send data to $j$. We define $C_{i j}$ as the following

$$
C_{i j}=R S d\left(x_{i}, x_{j}\right)^{\alpha},
$$

where $R S$ is a parameter depending on the transmitter/receiver antenna gains and the system loss not related to propagation, and $\alpha$ is the path loss exponent depending on the specific propagation environment.

Another example of cost in wireless communications is the data loss during transmission, which is related to the noise and interference of the wireless channel. Therefore, we can define $C_{i j}=h(N, I)>0$, where $N$ is the environment noise and $I$ is the interference.

\section{Social connection model}

We present a model used in social networks[5]. Assume that each node potentially offers benefits $V$ to other nodes per time unit. The potential benefit may be reduced with multi-hop interactions in the network. Following the JacksonWolinsky connections model, then the gain node $i$ obtains by connecting with node $j$ is defined as

$$
B_{i j}=\sum V \delta^{r_{i j}-1}
$$

where $r_{i j}$ is the number of hops in the shortest path between $i$ and $j$ (also known as the geodesic distance in graph theory), and $0 \leq \delta \leq 1$ is the communication depreciation rate. The gain function gives higher value to paths with smaller number of hops. It captures the fact that more directly collaborating nodes gain more than nodes far away in terms of 'social' distance. The depreciation can be explained by diminishing benefits due to intermediate collaboration failures or delays.

The cost can be the degree of trust between users $i$ and $j$ : the more $i$ trusts $j$, the lower the cost of establishing the link.

\section{CoAlition Formation Game}

We are interested in studying autonomous networks, where each node manages and controls its own operations in the network. A node decides to directly collaborate with others only if it gets the maximum payoff. The coalition formation process is modelled as pairwise games, which is called coalition formation game in the literature [6]. In this section, we give the detailed description of the games.

The pairwise games are modelled as an iterated process in which individual nodes activate and delete links based on the improvement that the resulting network offers them relative to the current network. A link between two nodes can be formed only if both nodes agree to activate the link, while a single node can sever an existing link. Each user receives a payoff based on the network configuration that is in place. 
Initially the $n$ nodes are disconnected. The players meet over time and have the opportunity to form links with each other. The time horizon, $T$, is divided into periods and is modelled as a countable, infinite set, $T=\{1,2, \ldots, t, \ldots\}$. Let $g^{(t)}$ represent the network that exists at the end of period $t$.

A strategy of node $i$ is a vector, defined as $\gamma_{i}=$ $\left(\gamma_{i, 1}, \ldots, \gamma_{i, i-1}, \gamma_{i, i+1}, \ldots, \gamma_{i, n}\right)$, where $\gamma_{i, j} \in\{0,1\}$ for each $j \neq i . \gamma_{i, j}=1$ is interpreted as saying that node $i$ wants to form a link with node $j$, while $\gamma_{i, j}=0$ states that $i$ does not directly communicate with node $j$. The set of all strategies of node $i$ is denoted by $\Gamma_{i}$. Since node $i$ has the option of forming or not forming a link with each of the remaining $n-1$ nodes, the number of strategies of node $i$ is $\left|\Gamma_{i}\right|=2^{n-1}$. The set $\Gamma=\Gamma_{1} \times \cdots \times \Gamma_{n}$ is the strategy space of all the nodes. A link $i j$ is formed in network $g$ only if $\gamma_{i, j}=1$ and $\gamma_{j, i}=1$. Therefore, a strategy profile $\gamma^{(t)}=\left(\gamma_{1}^{(t)}, \ldots, \gamma_{n}^{(t)}\right)$ at time period $t$ corresponds to the network $g^{(t)}$ at time $t$. The payoff of node $i$ from the network $g$ is defined as

$$
U_{i}(g)=B_{i}(g)-C_{i}(g),
$$

where $B_{i}(g)=\sum_{j \in N(g)} B_{i j}$ and $C_{i}(g)=\sum_{j \in N(g)} C_{i j}$.

Now we describe the dynamic process generated by the iterated pairwise game. The game is assumed to be repeated in each time period $t=1,2, \ldots$ Define $p_{i j}$ as the probability that the node pair $i j$ is selected, in each time period, to play the pairwise game. Notice that there may be multiple pairs selected in the same time period. These pairs can play simultaneously as long as they do not contain the same node. On the other hand, if both $i j$ and $i k$ are selected, $i$ cannot play two games simultaneously. Thus $i$ will not play any of the two games and it will inform its neighbors $j$ and $k$ as well. Therefore, there would be no game played on links $i j$ and $i k$ in the current time period.

We assume that each node is myopic. Given that nodes $i$ and $j$ play the game, if the link $i j$ is already in the network, then the decision is whether to sever it, and otherwise the decision is whether to establish the link. The nodes involved act myopically, activating the link if it makes each at least as well off and one strictly better off, and deleting the link if its deletion makes either player better off. Mathematically speaking, if only node pair $i j$ is selected in time period $t$, then the network $g^{(t+1)}$ has either

$$
\begin{array}{ll}
\text { - } & g^{(t+1)}=g^{(t)}-i j \text { if } v_{i}\left(g^{(t)}-i j\right)>v_{i}\left(g^{(t)}\right) \text { or } v_{j}\left(g^{(t)}-\right. \\
& i j)>v_{j}\left(g^{(t)}\right), \text { or } \\
\text { - } & g^{(t+1)}=g^{(t)}+i j \text { if } v_{i}\left(g^{(t)}+i j\right)>v_{i}\left(g^{(t)}\right) \text { and } v_{j}\left(g^{(t)}+\right. \\
& i j) \geq v_{j}\left(g^{(t)}\right) \text {, or } v_{i}\left(g^{(t)}+i j\right) \geq v_{i}\left(g^{(t)}\right) \text { and } v_{j}\left(g^{(t)}+\right. \\
& i j)>v_{j}\left(g^{(t)}\right) \text {, or } \\
\text { - } g^{(t+1)}=g^{(t)} \text { if none of the above satisfies. }
\end{array}
$$

If more than one pairs are selected to play the game, each pairwise game could be considered separately. If after some time period $t$, no additional links are formed or severed, then the network formation process has reached a steady state. Thus a coalition or coalitions have been formed at the steady state. Then the coalition formation game moves to the second phase, in which users act together to achieve maximum payoffs.

\section{GAME DYNAMICS}

In this section, we study the dynamics of the game we just defined, including its convergence, steady state and topology at the steady state.

\section{A. Convergence}

Having described the iterated pairwise game,we study the convergence of such a game. In particular, we are interested in the conditions under which all nodes in the network are connected. The coalition that contains all the nodes is called the "grand coalition".

To study the convergence, we first define a concept of stability: pairwise stability.

Definition 1 A network $g$ is pairwise stable if

- for all $i j \in g v_{i}(g) \geq v_{i}(g-i j)$ and $v_{j}(g) \geq v_{j}(g-i j)$, and

- for all $i j \notin g$, if $v_{i}(g)<v_{i}(g+i j)$ then $v_{j}(g)>v_{j}(g+$ $i j)$ or if $v_{j}(g)<v_{j}(g+i j)$ then $v_{i}(g)>v_{i}(g+i j)$.

We first give a simple fact on the dynamics of the pairwise game:

Lemma 1: The iterated pairwise game converges to a pairwise stable network or a cycle of networks.

Sketch of Proof: If in certain time period, the network is not pairwise stable, there must exist at least one link that can be formed or severed to improve the payoffs of the two end nodes. As long as such a link is selected, the network changes to another network. This procedure either stops at the pairwise stable network or it changes back to a network that has been met due to the limited number of possible networks $\left|g^{N}\right|$. In the later case, the procedure forms a cycle.

Figure 1(a) is a network of 6 nodes starting from no links. The benefit follows the definition of Eqn. (2). Take $c_{12}=$ $c_{23}=c_{34}=c_{45}=c_{56}=c_{61}=1$, where $c_{i j}=c_{j i}$ for all $i, j \in N$ and the cost of other links are much greater than $1, V=0.9$ and $\delta=0.3$. We observe that the first link's cost exceeds its payoff, while subsequent links are valuable. Following the myopic strategy, no link could be formed at all. However, it is obvious that the network shown in Figure 1(b) provides better payoffs than the empty network, where $v_{i}=$ 0.421 for all $i=1,2, \ldots, 6$, and it is easy to verify that the network is pairwise stable. Some random events are needed

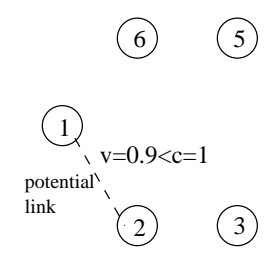

(a)

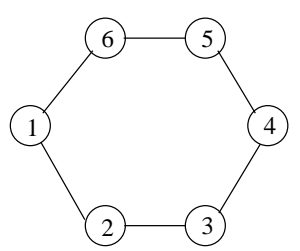

(b)
Fig. 1. A network where the game converges to an inefficient network. 
to help the network jump out of the inefficient stable network, which (random events) are called mutations.

In evolutionary games, mutations are introduced so that the evolution of games is modelled as a Markov chain, where the states of the Markov chain are the strategy profiles $\gamma$. Given nonzero mutations for each state of the Markov chain, we have that the Markov chain is irreducible and aperiodic. Therefore, it has a unique corresponding stationary distribution. The work of Harsanyi and Selten [7] and Kardori, et al. [8] show that by letting the mutation probability go to 0 in a certain way, the game converges to a unique Pareto equilibrium. The mutations for network formation mean that when two nodes agree to form a link, with a probability $p$, the link is not formed, or when a link is to be deleted because one of the two nodes choose to sever it, the link is not deleted with probability $p$. Such mutations may result from transmission failures or noise. Thus by using mutations, the pairwise game converges to a stable network.

The dynamic system defines a Markov chain on a finite state space. $P=\left[p_{i j}\right]$ is the Markov transition matrix. Note that, under our assumptions, all elements in the matrix $P$ are strictly positive. It is well known, then, that the Markov chain has a unique stationary distribution. A stationary distribution is a row vector $\mu$ satisfying

$$
\mu P=\mu .
$$

Asymptotically, independent of the initial condition, the strategy distribution is given by $\mu$. By introducing stochastic mutations, we have obtained uniqueness and global stability.

We examine the long run behavior of the system when the probability of mutations is small. To this end, we introduce the concept of the limit distribution.

Definition 2 The limit distribution $\mu^{*}$ is defined by $\mu^{*}=$ $\lim _{p \rightarrow 0} \mu(p)$, if it exists.

If the limit distribution places positive probability on the strategy configuration $z \in Z, z$ is called a long run equilibrium. In principle, we can calculate the limit distribution, for each $p$, by explicitly writing down the closed form for $p_{i j}$ and then solving the equation $\mu P=\mu$ for $\mu$, but the procedure is complicated. In this paper, we only present the result that shows that by permutation the game converges to a dominant equilibrium.

Theorem 2: If there exists an equilibrium which dominates other equilibria, with probability 1 the $N$-player game converges to the dominant equilibrium.

Proof: This is true since the dominant equilibrium is the single absorbing state. The Markov chain will converge to the single absorbing state with probability 1 .

\section{B. Steady state}

In this section, we investigate the coalitions formed when the pairwise games reach the steady state. The network model we studied is described as the following:

- Gain: $B_{i j}=B$ if $i j \in g$.

- The cost is the summation of two components: communication cost and risk that depends on trust values, $C_{i j}=C C_{i j}+R_{i j}$.

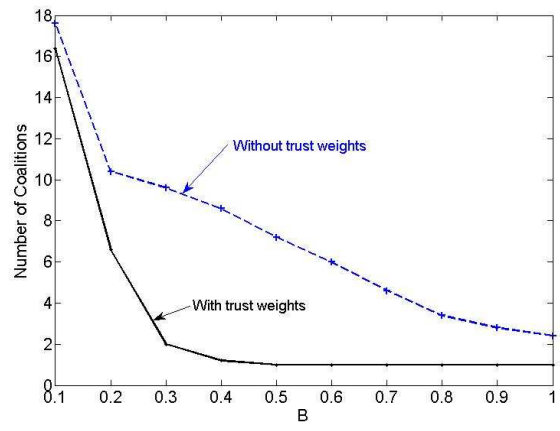

Fig. 2. Number of coalitions vs. benefit $B$

- The communication cost is defined as in Eqn. (1), $C C_{i j}=R S d_{i j}^{2}$, where $d_{i j}$ is the distance between $i$ and $j$.

- The risk is defined as the reverse of trust values. Trust values are based on the history of a node's game strategy. More specifically, if at time $T$, $s_{i j}(T)=\sum_{t=1}^{T} \gamma_{j i}(t) / T$, then $R_{i j}=1 / s_{i j}$.

- Game strategy: in addition to the strategy defined in Sec. III, a scheme that uses trust as an incentive for collaboration is introduced, where if $s_{i j}$ is less than a threshod $\theta$, node $i$ always chooses to collaborate with $j$.

We run simulations where 20 nodes are randomly placed on a 1000 meters $\times 1000$ meters square. Two nodes are selected to play the pairwise game with a fixed probability $1 / n(n-1)$, where $n=20$. Parameters $R S$ and $\theta$ are fixed and the benefit parameter $B$ changes. We compare the number of coalitions that are formed with and without the trust constraint when the network reaches the pairwise stable state as shown in Figure 2. It shows that the trust constraint serves as an incentive that helps the pairwise game to form the grand coalition (the number of coalitions is equal to 1 ).

\section{Topology Effects}

We studied the topology of the network when the game reaches the steady state. One particular topology we are interested in is the small-world topology: most links are connected between neighboring nodes with few long-range shortcuts. In the past five years, there has been substantial research on the small-world model in various complex networks, such as the Internet and biological systems. One important result is that the second largest eigenvalue modulus (SLEM) of the corresponding adjacency matrix of a graph determines the convergence speed of distributed dynamic algorithms on the graph.

In the rest of this section, we investigate the normalized matrix $F$ of the adjacency matrix $A$, that is

$$
F=D^{-1} A,
$$

where $D$ is the diagonal matrix whose $i$ th diagonal element is the degree of vertex $i$. Thus $F$ matrices are a class of stochastic matrices. Since $F$ is a primitive stochastic matrix, according to the Perron-Frobenius theorem [9], $\lambda_{1}$ 


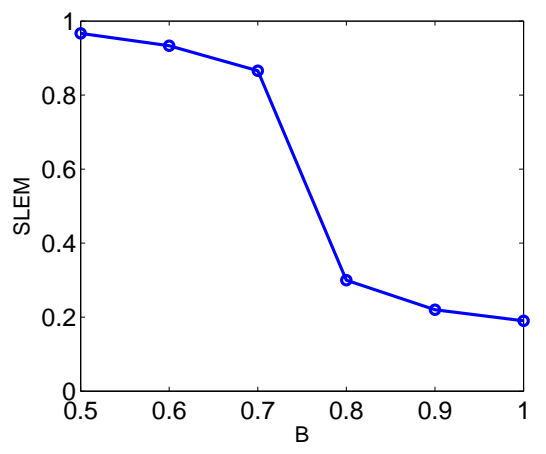

Fig. 3. SLEM vs. benefit $B$

is a simple eigenvalue with a right eigenvector $\mathbf{1}$ and a left eigenvector $\pi$ such that $\mathbf{1}^{T} \pi=1$. Let $\lambda_{2}, \lambda_{3}, \ldots, \lambda_{r}$ be the other eigenvalues of $F$ ordered in a way such that $\lambda_{1}=1>\left|\lambda_{2}\right| \geq\left|\lambda_{3}\right| \geq \cdots \geq\left|\lambda_{r}\right|$, and $m_{2}$ is the algebraic multiplicity of $\lambda_{2}$. Then it is shown that the convergence of $F^{t}$ is geometric, with relative speed equal to SLEM $=\left|\lambda_{2}\right|$. The spectral gap of a graph is the quantity 1 - SLEM; so iterations on graphs with higher spectral gaps converge faster.

We study the SLEM of graphs that are formed by the formation game we described in Sec. IV-B. Since the SLEM is defined in a connected graph, we only study benefit parameter values $B>0.5$, when a grand coalition is formed at the steady state as shown in Fig 2. In Figure 3, the SLEM decreases with the benefit. Especially, the SLEM decreases dramatically in the range of $[0.7,0.8]$. We calculated the shortest path and clustering coefficients of these graphs. The results show that the range of $[0.7,0.8]$ is exactly where we observe the small-world property. Research has shown that a graph with the small-world property is efficient regarding convergence of distributed algorithms. Our study on network structure formation shows that a small increase in benefit may result in an efficient network topology.

More specifically, we study networks in the form similar to the one of Fig. 1, that is, all nodes are placed on the circumference of a circle. Suppose that the gain and cost are defined as in Eqns. (2) and (1) respectively. We assume that all nodes are equally placed on the circle. Therefore, the distance between any pair of neighboring nodes on the circle is the same, denoted as $d_{1}$, and the corresponding cost is $C_{d_{1}}$. Similarly, the distance between nodes that are $r$ hops away on the circle is denoted as $d_{r}$ and the corresponding cost is $C_{d_{r}}$.

We first present the following proposition:

Proposition 3: There exist direct connections between nodes that are at least $r$ hops away on the circle if $V>$ $\frac{C_{d_{r}}}{1-\delta\left\lfloor\frac{n}{2}\right\rfloor-r+1}$.

Sketch of Proof: Suppose nodes are numbered in a clockwise order. Now consider a connection that connects nodes 1 and $r+1$. By adding this connection, the benefit of node 1 from node $r+1$ to node $\left\lfloor\frac{n}{2}\right\rfloor$ changes to

$$
V\left(1+\delta+\cdots+\delta^{\left\lfloor\frac{n}{2}\right\rfloor-r}\right)-C_{d_{r}} .
$$

The least gain change due to adding the connection between node 1 and $r+1$ takes place if there has been a connection between node 1 and $r$. Therefore, a direct connection that connects nodes 1 and $r+1$ is added if

$V\left(1+\delta+\cdots+\delta^{\left\lfloor\frac{n}{2}\right\rfloor-r}\right)-C_{d_{r}}>V\left(\delta+\cdots+\delta^{\left\lfloor\frac{n}{2}\right\rfloor-r+1}\right)$.

The right hand side is the gain node 1 gets from node $r$ to $\left\lfloor\frac{n}{2}\right\rfloor$ given there is a direct connection between node 1 and $r-1$ and no direct connection between node 1 and $s$, where $s \geq r$.

Then we have that

$$
V>\frac{C_{d_{r}}}{1-\delta\left\lfloor\frac{n}{2}\right\rfloor-r+1} .
$$

Notice that the condition on $V$ is only a sufficient condition.

Consider the basic case where the formed network is the 1-dimensional lattice having a ring topology. We have that

Corollary 4: The formed network is a 1-dimensional lattice if $C_{d_{1}}<V<\frac{C_{d_{2}}}{1-\delta\left\lfloor\frac{n}{2}\right\rfloor-1}$.

The corresponding $F$ matrix of the network is called the base matrix $F_{0}$ and is the following:

$$
F_{0}=\left(\begin{array}{cccccc}
0 & \frac{1}{2} & 0 & \ldots & 0 & \frac{1}{2} \\
\frac{1}{2} & 0 & \frac{1}{2} & 0 & \ldots & 0 \\
0 & \frac{1}{2} & 0 & \frac{1}{2} & \ldots & 0 \\
. & . & . & . & . & . \\
. & . & . & . & . & . \\
\frac{1}{2} & 0 & 0 & \ldots & \frac{1}{2} & 0
\end{array}\right)
$$

The matrix $F_{0}$ is actually a circulant matrix. Circulant matrices have a special structure which provides them with special properties. All entries in a given diagonal are the same. Each row is determined by its previous row by a shift to the right (modulo $n$ ). Suppose the first row of a circulant matrix is $\left[a_{1}, a_{2}, \ldots, a_{n}\right]$. We write a circulant matrix $A=\operatorname{circ}\left[a_{1}, a_{2}, \ldots, a_{n}\right]$. Now consider the $n \times n$ permutation matrix, $\Pi=\operatorname{circ}[0,1,0, \ldots, 0]$. Then $A$ can be written as $A=a_{1} I+a_{2} \Pi+\cdots+a_{n} \Pi^{n-1}$. For a vector $a=\left[a_{1}, a_{2}, \ldots, a_{n}\right]$, the polynomial $p_{a}(z)=a_{1}+a_{2} z+$ $\cdots+a_{n} z^{n-1}$ is called the representer of the circulant. The following theorem based on [10] states how to calculate the eigenvalues of circulants.

Theorem 5: [10] Let $\omega=e^{\frac{2 \pi \sqrt{-1}}{n}}$ be the $n$th root of unity. The eigenvalues of $A=\operatorname{circ}\left[a_{1}, a_{2}, \ldots, a_{n}\right]$ are given by $\lambda_{i}=p_{a}\left(\omega^{i-1}\right)$, where $i=1,2, \ldots, n$.

Then we have the following proposition:

Proposition 6: The SLEM of the base matrix $F_{0}$ has multiplicity at least 2 .

Sketch of Proof: $\quad F_{0}$ is a circulant matrix. The representer of the circulant $F_{0}$ is

$$
p_{a}(z)=\frac{1}{2}\left(z+z^{n-1}\right) .
$$


So the eigenvalues of this matrix are $\lambda_{i}=p_{a}\left(\omega^{i-1}\right)$. It is easy to show that $\lambda_{1}=1$ and more over it is a simple eigenvalue because the underlying graph is connected. Since for integers $A$ and $B, \omega^{A n+B}=\omega^{B}$, it follows that $\lambda_{2}=\lambda_{n}$, $\lambda_{3}=\lambda_{n-1}$ and so on.

By substituting $\lambda_{2}=p_{a}(\omega)$, we have that

Corollary 7: $\operatorname{SLEM}\left(F_{0}\right)=\cos \left(\frac{2 \pi}{n}\right)$.

Now consider the case where $V>\frac{C_{d_{r}}}{1-\delta^{\left\lfloor\frac{n}{2}\right\rfloor-r+1}}$, where $r$ is any integer in the range $\left[2,\left\lfloor\frac{n}{2}\right\rfloor\right]$. Shortcuts with length at least $r$ (i.e. direct connections among nodes $r$ hops away) are added in the network. The larger $V$ is, the 'longer' shortcuts are established.

We investigate the effect of shortcuts following the perturbation approach to small worlds proposed by Higham [11]. We add "small" nonzero positive numbers for the entries of $F_{0}$ that correspond to connections between nodes that are at most $r$ hops away. We perturb these entries of the matrix $F_{0}$ by a constant $\varepsilon$. The idea is that $\varepsilon$ represents the probability of having a shortcut. Then the expected number of shortcuts added is $\varepsilon(r-1) n$. This model can be considered as a "mean field" approximation of the real network. We anticipate that the analysis of these perturbed matrices gives us some insight on the evolution of network topologies as the gain increases.

The perturbed matrix is the following:

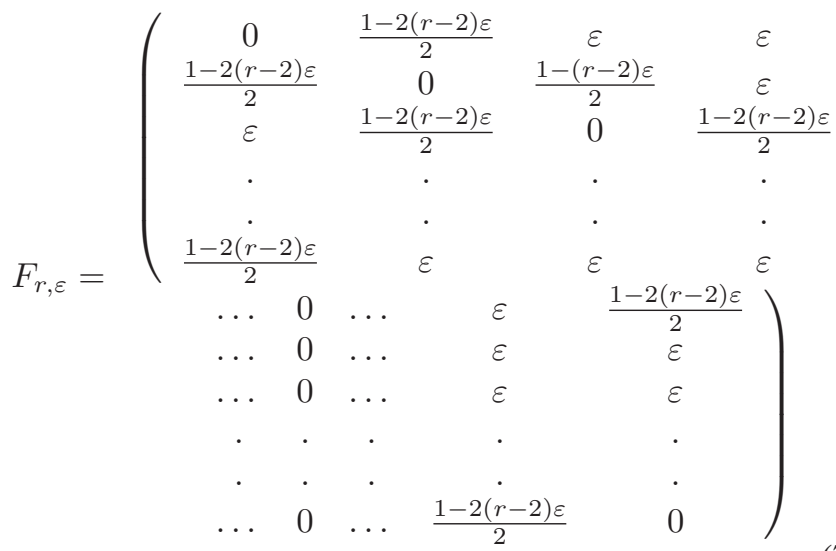

$F_{r, \varepsilon}$ is also a circulant matrix. The representer of this circulant is

$$
\begin{aligned}
p_{a}(z)= & \frac{1-2(r-2) \varepsilon}{2} z+\varepsilon z^{2}+\cdots+\varepsilon z^{r-1}+0+\cdots+0 \\
& +\varepsilon z^{n-r+1}+\cdots+\varepsilon z^{n-2}+\frac{1-2(r-2) \varepsilon}{2} z^{n-1} .(8)
\end{aligned}
$$

Substituting $z=\omega$, we have the SLEM of $F_{r, \varepsilon}$

$$
\lambda_{2}\left(F_{r, \varepsilon}\right)=(1-2(r-1) \varepsilon) \cos \left(\frac{2 \pi}{n}\right)+2 \varepsilon \sum_{k=1}^{r-1} \cos \left(\frac{2 k \pi}{n}\right) .
$$

Thus we can state the following proposition:

Proposition 8: Let $r \varepsilon=\frac{K}{n^{\beta}}$, where $K>0$ and $\beta \geq 0$. For $\beta>2$, the effect of shortcuts on convergence rate is negligible. $\beta=2$ is the threshold. For $\beta<2$, the shortcuts are dominantly decreasing the SLEM, thus the small-world topology appears.
Proof: For large $n$, applying Taylor series, we can write:

$$
\lambda_{2}\left(F_{r, \varepsilon}\right)=1-\frac{2 \pi^{2}}{n^{2}}-2 r \varepsilon+o\left(\frac{1}{n^{4}}\right) .
$$

The first two terms are the contributions of the base matrix $F_{0}$ and the rest are the contributions of the perturbation. It is easy to yield the results of this proposition.

\section{CONLUSions}

In this paper, we developed the fundamental view that agents in an autonomic network collaborate to accomplish objectives and goals much better than working alone, or even accomplish objectives that they cannot achieve alone at all. We modelled the process of coalition formation by understanding and quantifying the tradeoff between the benefits vs. the costs of collaboration. Our analysis leads to new methods that can be used to design and control/operate networks of agents. Multiple metrics for benefits and costs can be considered within this framework including trust values between agents. Furthermore, we investigated the topology effects of formed coalitions by studying the graph's spectral gap. Our future work will integrate the current framework into real networks, such as communication networks and biological networks, and study how the new methods can be applied to fundamental network design.

\section{ACKNOWLEDGMENTS}

This work was prepared through collaborative participation in the Communications and Networks Consortium sponsored by the U.S. Army Research Laboratory under the Collaborative Technology Alliance Program, Cooperative Agreement DAAD19-01-2-0011.

\section{REFERENCES}

[1] N. R. C. for the National Academies, Network Science. The National Academies Press, 2005.

[2] — Strategy for an Army Center for Network Science, Technology, and Experimentation. The National Academies Press, 2007.

[3] J. S. Baras and T. Jiang, "Cooperation, trust and games in wireless networks," in Proceedings of Symposium on Systems, Control and Networks, honoring Professor P. Varaiya. Birkhause, June 2005, pp. 183-202.

[4] T. Jiang and J. S. Baras, "Trust evaluation in anarchy: A case study on autonomous networks," in Proceedings of 2006 INFOCOM, Barcelona, Spain, April 2006.

[5] M. O. Jackson and A. Wolinsky, "A strategic model of social and economic networks," Journal of Economic Theory, vol. 71, no. 1, pp. 44-74, October 1996.

[6] M. Slikker and A. V. d. Nouweland, Social and Economic Networks in Cooperative Game Theory, ser. SERIES C: GAME THEORY, MATHEMATICAL PROGRAMMING AND OPERATIONS RESEARCH. Kluwer Academic Publishers, 2001, vol. 27.

[7] J. C. Harsanyi and R. Selten, A General Theory of Equilibrium in Games. Cambridge MIT Press, 1988.

[8] M. Kandori, G. J. Mailath, and R. Rob, "Learning, mutation, and long run equilibria in games," Econometrica, vol. 61, no. 1, pp. 29-56, 1993.

[9] E. Seneta, Non-negative matrices and Markov chains, 2nd ed., ser. Springer series in statistics. Springer-Verlag New York Inc., 1981.

[10] P. Davis, Circulant Matrices. Wiley, 1979.

[11] D. J. Higham, "A matrix perturbation view of the small world phenomenon," SIAM Journal on Matrix Analysis and Applications, vol. 25, no. 2, pp. 429-444, 2003. 